\title{
Activation of the endoplasmic reticulum stress pathway involving CHOP in the lungs of rats with hyperoxia-induced bronchopulmonary dysplasia
}

\author{
HONG-YAN LU，JIE ZHANG，QIU-XIA WANG，WEI TANG and LU-JIE ZHANG \\ Department of Pediatrics, The Affiliated Hospital of Jiangsu University, Zhenjiang, Jiangsu 212001, P.R. China
}

Received September 12, 2014; Accepted May 28, 2015

DOI: $10.3892 / \mathrm{mmr} .2015 .3979$

\begin{abstract}
The molecular pathomechanisms underlying bronchopulmonary dysplasia (BPD) remain to be fully elucidated, however, lung injury is considered to be a key event. The present study was performed to determine the role of endoplasmic reticulum (ER) stress and investigate the apoptosis of alveolar epithelial cells in a BPD rat model. A total of 48 preterm Sprague-Dawley rats were randomly divided into a control group and a hyperoxia group. The rats in the BPD group were exposed to $85 \%$ hyperoxia, while the rats in the control group were exposed to room air. A total of eight rats in each group were sacrificed 7, 14 or 21 days after exposure. The expression levels of 78-kDa glucose-regulated/binding immunoglobulin protein (GRP78) and CCAAT/enhancer binding protein homologous protein (CHOP) in the lung tissues were examined using immunohistochemistry, and the mRNA and protein levels of GRP78 and CHOP were detected using reverse transcription-quantitative polymerase chain reaction and western blot analyses, respectively. In addition, the levels of apoptosis in the lung cells were evaluated suing terminal deoxynucleotidyl transferase-mediated dUTP nick-end labeling. It was demonstrated that the mRNA and protein levels of GRP78 and $\mathrm{CHOP}$, and the levels of cell apoptosis in the hyperoxia group differed significantly from those in the control group $(\mathrm{P}<0.05)$ at different time-points, and increased with extension of the duration of hyperoxic exposure. These data demonstrated that the ER stress pathway, involving CHOP, is activated and is important in the pathogenesis of BPD.
\end{abstract}

Correspondence to: Dr Hong-Yan Lu, Department of Pediatrics, The Affiliated Hospital of Jiangsu University, 438 Jiefang Road, Zhenjiang, Jiangsu 212001, P.R. China

E-mail: 1hy5154@163.com

Key words: CCAAT/enhancer binding protein homologous protein, endoplasmic reticulum stress, apoptosis, bronchopulmonary dysplasia

\section{Introduction}

Oxygen supplementation is a common clinical intervention for premature infants with respiratory failure, however, prolonged exposure to hyperoxic conditions has long been recognized as a potential risk factor for bronchopulmonary dysplasia (BPD) (1). Exposing premature newborn rats to high concentrations of ambient oxygen induces the enlargement of distal air spaces with simplification of lung structure, which recapitulates the histopathology of BPD (2). Hyperoxia therapy produces reactive oxygen species (ROS), which can induce cell death, disrupt postnatal alveolarization, lead to abnormal lung repair and cause lung tissue injury (3). Although the specific mechanisms by which hyperoxia causes lung injury remain to be fully elucidated, the generation of ROS and cellular apoptosis appear to be important.

Increasing evidence has indicated that endoplasmic reticulum (ER) stress is involved in apoptosis. The ER is an important organelle in mammalian cells with numerous functions, including the maintenance of intracellular calcium homeostasis, protein secretion, lipid synthesis, and protein glycosylation and folding (4). Interruption of ER homeostasis leads to ER stress and triggers the unfolded protein response (UPR), resulting in activation of three ER stress receptors, inositol requiring protein-1, protein kinase RNA-like ER kinase and activating transcription factor 6 , which result in apoptosis in persistently stressed cells $(5,6)$.

The 78-kDa glucose-regulated/binding immunoglobulin protein (GRP78) is a hallmark of the UPR and ER stress responses (6). The UPR responds rapidly to ER stress to enhance cell survival. However, if protein aggregation is persistent and stress cannot be resolved, signaling switches from the pro-survival to the pro-apoptotic ER stress response $(5,7,8)$. Evidence has suggested that CCAAT/enhancer binding protein homologous protein (CHOP) is recruited and is involved in ER stress-induced apoptosis, following the phosphorylation of eukaryotic initiation factor $2 \alpha$ and upregulation of activating transcription factor $4(6,9,10)$. CHOP is a $29 \mathrm{kDa}$ protein with 169 (human) or 168 (rodent) amino-acid residues. CHOP is also termed growth arrest, DNA damage inducible gene 153 and DNA damage-inducible transcript 3 (11). The induction of CHOP may trigger ER stress-induced apoptosis, and the involvement of CHOP-mediated apoptosis has been demonstrated in various diseases, including diabetes, 
neurodegenerative diseases, brain ischemia and certain cardiovascular diseases (12-15). However, the importance of ER stress and the role of CHOP in the pathogenesis of neonatal lung injury and hyperoxia-induced BPD remain to be fully elucidated. The present study hypothesized that hyperoxia-induced BPD results from persistent ER stress, leading to increased expression of CHOP and cell death.

\section{Materials and methods}

Study animals and procedures. Adult Sprague-Dawley (SD) rats, including 21 females (200-220 g) and seven males (220-250 g) were purchased from the Experimental Animal Center of Jiang Su University (Zhenjiang, China). The rats were maintained under controlled temperature $\left(23 \pm 1^{\circ} \mathrm{C}\right)$, humidity, and lighting ( $12 \mathrm{~h}$ light/12 h dark cycle), and were fed a standard rodent chow diet. All procedures and protocols were reviewed and approved by the Animal Care and Ethics Committee of Jiang Su University.

Hyperoxia exposure. The rats were left overnight for mating in groups of four consisting of three females and one male. The day on which the vaginal plug was identified was considered to be the first day of pregnancy. On the 21st day of pregnancy (term $=22$ days), the pregnant rats were anesthetized with intraperitoneal injections of $10 \%$ chloral hydrate $(350 \mathrm{mg} / \mathrm{kg}$; Wuhan Boster Biological Technology, Ltd., Wuhan, China), and then underwent delivery via uterine-incision, resulting in pups deemed premature. Within $24 \mathrm{~h}$ of birth, the newborn rats were placed in chambers in which the oxygen concentration was maintained at $\mathrm{FiO}_{2}=0.85$ for 7,14 and 21 days, or were maintained in standard room air in which the oxygen concentratoin was maintained at $\mathrm{FiO}_{2}=0.21$. The nursing dams were provided with food and water ad libitum, were maintained on a 12:12-h on-off light cycle and were fostered by rotating in and out of the chamber every $24 \mathrm{~h}$ to avoid oxygen toxicity. The oxygen concentration was monitored continuously using an oxygen analyzer (model 572; Servomex Co., Inc., Norwood, MA, USA).

Lung histology and morphometric analysis. Following continued exposure to oxygen $(n=24)$ or room air $(n=24)$ for 7, 14 and 21 days, the premature rats were sacrificed with intraperitoneal injections of pentobarbital $(100 \mathrm{mg} / \mathrm{kg}$; Tianjin Pharmaceutical Group Xinzheng Co., Ltd., Xinzheng, China). A catheter was placed into the trachea and the lungs were inflated and maintained at $30 \mathrm{~cm} \mathrm{H}_{2} \mathrm{O}$ pressure with $4 \%$ paraformaldehyde in phosphate-buffered saline (PBS) for $45 \mathrm{~min}$. A ligature was tightened around the trachea to maintain pressure following removal of the tracheal cannula. The lungs were removed and immersed in paraformaldehyde solution overnight, and the left lower lobes were embedded in paraffin. Sections were then cut using a microtome set at $5 \mu \mathrm{m}$ and mounted on RNase free slides for hematoxylin and eosin (HE) staining, immunohistochemistry and a terminal deoxynucleotidyl transferase-mediated dUTP-biotin nick end labeling (TUNEL) assay. At least three lung sections from each animal were subjected to morphometric analysis. Alveolarization was assessed by performing radial alveolar counts (RACs), according to the method of Emery and Mithal (16). From the center of the respiratory bronchiole, a
Table I. Sequence for each primer and product length.

\begin{tabular}{llr} 
Gene & \multicolumn{1}{c}{ Primer sequence $\left(5^{\prime}-3^{\prime}\right)$} & $\begin{array}{c}\text { Length } \\
(\mathrm{bp})\end{array}$ \\
\hline \multirow{2}{*}{ GRP78 } & $\begin{array}{l}\text { F CCGTAACAATCAAGGTCTACGA } \\
\text { R AAGGTGACTTCAATCTGGGGTA }\end{array}$ & 124 \\
& $\begin{array}{l}\text { RHOP } \\
\text { F ACCACCACACCTGAAAGCA }\end{array}$ & 86 \\
\multirow{3}{*}{-actin } & R AGCTGGACACTGTCTCAAAGG & \\
& F GCAGAAGGAGATTACTGCCCT & 136 \\
& R GCTGATCCACATCTGCTGGAA & \\
\hline
\end{tabular}

Primer sequence and product length designed for GRP78, CHOP and $\beta$-actin. GRP78, 78-kDa glucose-regulated/binding immunoglobulin protein; CHOP, CCAAT/enhancer binding protein homologous protein; F, forward; R, reverse.

perpendicular was drawn to the edge of the acinus, defined by a connective tissue septum or the pleura, and the number of septa intersected by this line were counted. A total of six counts were performed for each animal.

Reverse transcription-quantitative polymerase chain reaction $(R T-q P C R)$. The total RNA was extracted from the frozen lung tissues using TRIzol reagent (Invitrogen Life Technologies, Carlsbad, CA, USA) according to the manufacturer's instructions. The concentration of total RNA was quantified using spectrophotometry (Bio-Rad Laboratories, Inc., Hercules, CA, USA). RT was performed to synthesize the first-strand cDNA using a M-MLV Reverse Transcriptase system (Promega Corporation, Madison, WI, USA) and oligo (dT). The resulting cDNA was then subjected to RT-qPCR for evaluation of the relative mRNA levels of GRP78, CHOP and $\beta$-actin (internal control). The sequences for each primer (Takara Biotechnology Co., Ltd., Dalian, China) and product are shown in Table I. Gene-specific amplification was performed using a $20 \mu \mathrm{l} \mathrm{PCR}$ mix containing $1 \mu \mathrm{l}$ cDNA, $10 \mu \mathrm{l}$ of $2 \mathrm{X}$ SYBR Green master mix (Invitrogen Life Technologies) and $200 \mathrm{nmol}$ sense and antisense primers. The mix was preheated at $95^{\circ} \mathrm{C}(5 \mathrm{~min})$ and then amplified at $95^{\circ} \mathrm{C}(15 \mathrm{sec})$ and $60^{\circ} \mathrm{C}(20 \mathrm{sec})$ for 45 cycles. The relative expression levels of GRP78 and CHOP mRNA were determined by comparing with standard curves and normalization to $\beta$-actin. The experiment was performed in triplicate.

Western blot analysis. The homogenized lung tissue samples, were lysed in ice-cold isolation solution containing $250 \mathrm{mmol} / \mathrm{l}$ sucrose, $10 \mathrm{mmol} / \mathrm{l}$ triethanolamine, $1 \mu \mathrm{g} / \mathrm{ml}$ leupeptin and $0.1 \mathrm{mg} / \mathrm{ml}$ phenylmethylsulfonyl fluoride, and harvested following centrifugation $(12,000 \mathrm{xg})$ at $4^{\circ} \mathrm{C}$ for $30 \mathrm{~min}$. The protein samples $(\sim 40 \mu \mathrm{g})$ were then separated by electrophoresis on a $12 \%$ sodium dodecyl sulfate polyacrylamide gel and transferred onto polyvinylidene fluoride membranes (EMD Millipore, Bedford, MA, USA). Following blocking of the non-specific binding sites for 60 min with $5 \%$ non-fat milk, the membranes were incubated with primary antibodies overnight at $4^{\circ} \mathrm{C}$. The following primary polyclonal rabbit anti-rat antibodies were used: GRP78 (1:200; cat. no. sc-13968; Santa 
Cruz Biotechnology, Inc., Santa Cruz, CA, USA), CHOP (1:500; cat. no. sc-793; Santa Cruz Biotechnology, Inc.) and $\beta$-actin (1:1,000; cat. no. sc-1616-R; Santa Cruz Biotechnology, Inc.). The membranes were then washed three times in Tris-buffered saline with $0.1 \%$ Tween 20 (TBST) for $10 \mathrm{~min}$ and incubated with biotinylated goat anti-rabbit immunoglobulin G (1:200; Wuhan Boster Biological Technology, Ltd.) in PBS for $2 \mathrm{~h}$ at room temperature, followed by washing in TBST three times, for $5 \mathrm{~min}$ each. The membranes were then incubated for $1 \mathrm{~min}$ using an enhanced chemiluminescence system (Applygen Technologies, Inc., Beijing, China). An autoradiograph was obtained with an exposure time of 2-5 min. The relative band intensity was scanned and quantified using a GDS-800 Complete Gel Documentation \& Analysis system (Microtek Lab, Inc., Carson, CA, USA).

Immunohistochemical staining. Immunohistochemical staining was performed on tissues at 14 days after hyperoxia or air exposure. Following deparaffinization, endogenous peroxidase activity was quenched with $3 \%$ hydrogen peroxide. Subsequently, the slides were boiled in citrate buffer in a microwave for $15 \mathrm{~min}$. Following blocking of nonspecific binding using 5\% bovine serum albumin (Wuhan Boster Biological Technology, Ltd.), the slides were incubated with GRP78 antibody (1:150) or CHOP antibody (1:200) overnight at $4^{\circ} \mathrm{C}$, and then washed three times with PBS for $5 \mathrm{~min}$. The sections were then incubated for $1 \mathrm{~h}$ with a 500 -fold dilution of secondary antibodies conjugated with peroxidase, and incubated with 0.05\% 3,3'-diaminobenzidine (DAB; Wuhan Boster Biological Technology, Ltd.) solution for visualization. Finally, staining was performed using hematoxylin for $3 \mathrm{sec}$ and the slides were visualized under microscopy (BX51; Olympus Corporation, Tokyo, Japan).

Detection of apoptosis. The present study investigated whether hyperoxic exposure induces apoptosis in lung cells. Apoptosis was detected using a TUNEL assay 14 days after hyperoxia or air exposure, according to the manufacturer's instructions (Roche Applied Science, San Francisco, CA, USA). Briefly, the sections were deparaffinized, digested with proteinase $\mathrm{K}$ $(1: 200)$ at $37^{\circ} \mathrm{C}$ for $10 \mathrm{~min}$, and soaked in PBS for $5 \mathrm{~min}$. Each section was covered with a terminal deoxynucleotidyl transferase (TDT) enzyme solution, containing $45 \mu$ l equilibration buffer, $1 \mu 1$ biotin-11-dUTP and $4 \mu 1$ TDT enzyme, and incubated for $2 \mathrm{~h}$ at $37^{\circ} \mathrm{C}$ in a humidified chamber. The sections were immersed in stop buffer to terminate the enzymatic reaction and gently rinsed with PBS, followed by incubation with biotinylated anti-digoxin antibody $(1: 200)$ at $37^{\circ} \mathrm{C}$ for $30 \mathrm{~min}$, $\mathrm{SABC}(1: 100)$ at $37^{\circ} \mathrm{C}$ for $30 \mathrm{~min}, \mathrm{DAB}$ coloration, and visualization using microscopy (BX51; Olympus Corporation). Sections incubated with PBS, instead of TDT enzyme solution served as the negative controls. The TUNEL-positive cells were counted in six randomly selected visual fields at the same magnification in the control and treated mice (magnification, $\mathrm{x} 400$ ). The percentage of apoptotic cells was determined as the percentage of the total cells positive for TUNEL.

Statistical analysis. All data are expressed as the mean \pm standard deviation and were analyzed using SPSS 13.0 (SPSS, Inc., Chicago, IL, USA). Differences among groups were assessed

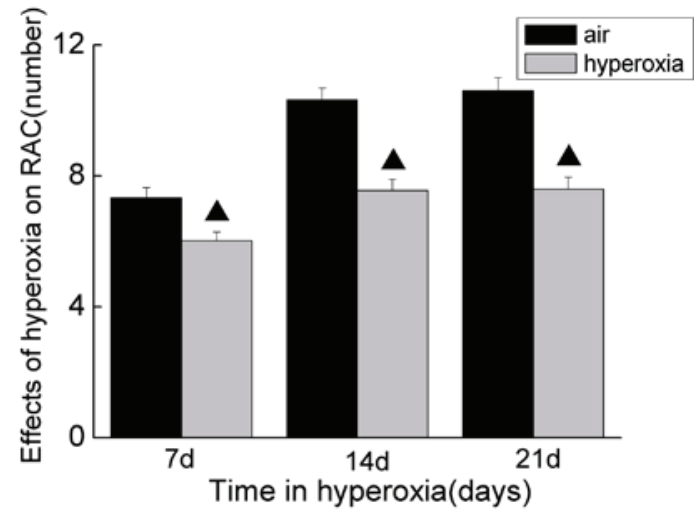

Figure 1. Effects of hyperoxia on lung histology and RAC. Compared with the control rats, the lung histology of the rats exposed to hyperoxia was characterized by decreased septation, distal air space enlargement and a reduction in complexity. The lung histology of the neonatal rats exposed to hyperoxia also exhibited a pattern of alveolar simplification in the 14 and 21-day-old rats. The alveolar number was determined by the RAC, which decreased following exposure to hyperoxia. Data are expressed as the mean \pm standard deviation. ${ }^{\wedge} \mathrm{P}<0.05$, compared with the control. RAC, radial alveolar counts.
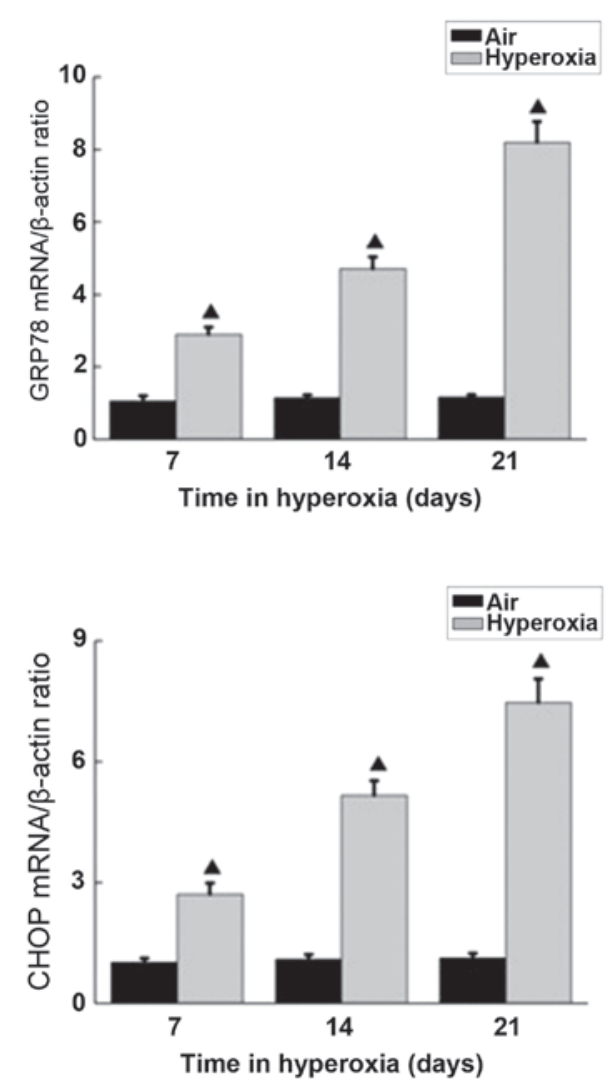

Figure 2. Effects of hyperoxia on the mRNA expression levels of GRP78 and CHOP. Total RNA was extracted from the lung tissues and subjected to reverse transcription-quantitative polymerase chain reaction. Following exposure of the rats to $85 \%$ hyperoxia, the mRNA expression levels of GRP78 and CHOP increased gradually between 7 and 21 days, and the expression levels were significantly higher, compared with the control group. Data are expressed as the mean \pm standard deviation. ${ }^{\boldsymbol{\Delta}} \mathrm{P}<0.05$, compared with control. GRP78, 78-kDa glucose-regulated/binding immunoglobulin protein; CHOP, CCAAT/enhancer binding protein homologous protein.

using a one-way analysis of variance and between pairs using Student's t-test. $\mathrm{P}<0.05$ was considered to indicate a statistically significant difference. 

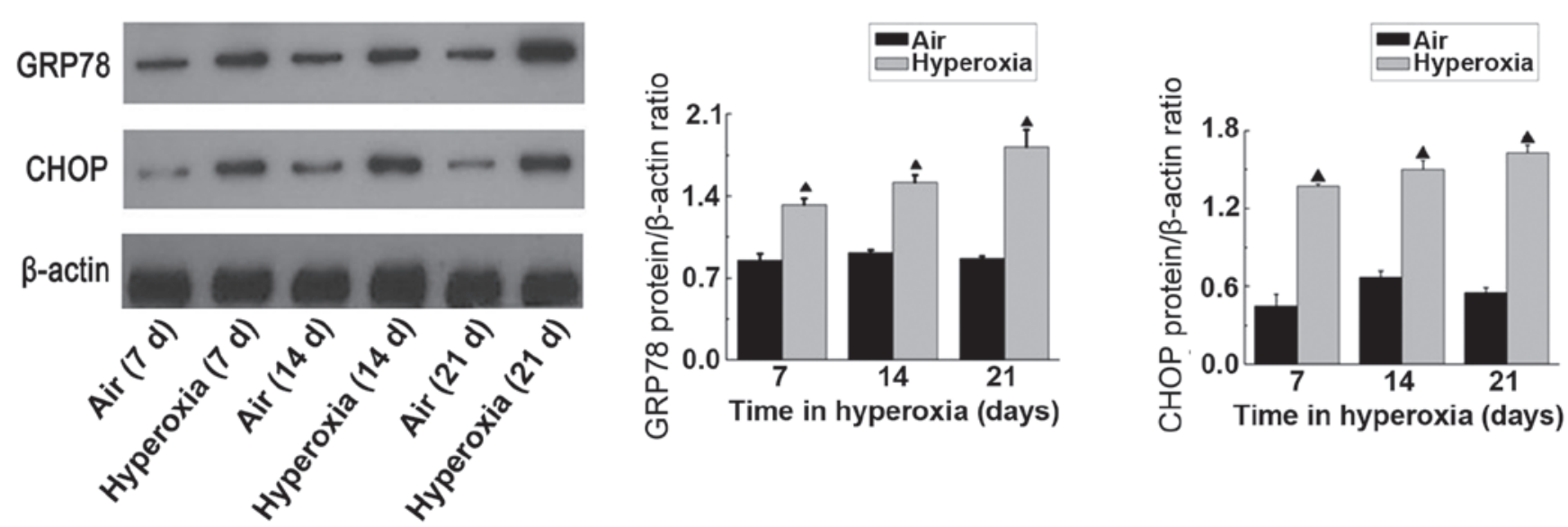

Figure 3. Protein levels of GRP78 and CHOP, determined using western blot analysis. Premature rats were exposed to $85 \%$ oxygen for 7 , 14 or 21 days. The results revealed an increase in the protein levels of GRP78 and CHOP in the lung tissues from the rats exposed to hyperoxia, compared with the rats exposed to normal air. Data are expressed as the mean \pm standard deviation. ${ }^{\mathbf{\Delta}} \mathrm{P}<0.05$, compared with the control. GRP78, 78-kDa glucose-regulated/binding immunoglobulin protein; CHOP, CCAAT/enhancer binding protein homologous protein.

\section{Results}

Lung histology and RAC. Compared with the control rats, the lung histology of the rats exposed to hyperoxia was characterized by decreased septation, distal air space enlargement and a reduction in complexity. To quantitfy the apparent decreases in the number of alveoli, RACs were performed. As shown in Fig. 1, the RACs were lower in the rats exposed to hyperoxia, compared with the rats exposed to room air. The neonatal rats exposed to hyperoxia exhibited a histological pattern of alveolar simplification and a reduced RAC, which was similar to the histology observed in human infants with BPD (17). These results demonstrated the animal model of BPD was successful.

mRNA expression levels of GRP78 and CHOP. RT-qPCR was performed on the rat lungs exposed to hyperoxia and room air to determine the mRNA levels of GRP78 and CHOP. As shown in Fig. 2, the mRNA expression levels of GRP78 and $\mathrm{CHOP}$ in the room air and hyperoxia-exposed rat lungs were detectable, and no differences in expression were observed in the lungs of the control rats over the entire 21 day time-course. However, in the rats were exposed to $85 \%$ hyperoxia, the mRNA expression levels of GRP78 and CHOP increased gradually over 7, 14 and 21 days, and the expression levels were significantly higher than those in the control group. In addition, as the duration of hyperoxia exposure increased, the increased differences between the two groups became more significant (Fig. 2). These data demonstrated that hyperoxia had similar effects on the expression levels of GRP78 and $\mathrm{CHOP}$, contributing to lung injury in BPD rats.

Expression of GRP78 and CHOP, determined using western blot analysis. The protein levels of GRP78 and CHOP were determined using western blot analysis. To determine whether hyperoxia induced the expression of CHOP and GRP78, the newborn rats were exposed to $85 \%$ oxygen for 7,14 or 21 days. The results of the western blot analysis revealed a GRP78 band at $78 \mathrm{kDa}$ and a $\mathrm{CHOP}$ band at $30 \mathrm{kDa}$. Consistent with the RT-qPCR results, increases in the protein expression levels of GRP78 and CHOP were observed in the lungs from the rats exposed to hyperoxia, compared with the age-matched controls exposed to normal air (Fig. 3). Over the time-course of hyperoxic exposure, the protein levels of GRP78 and CHOP gradually increased. These data demonstrated that GRP78 and CHOP were activated in the hyperoxia-exposed newborn rat lungs, further supporting the hypothesis that hyperoxia leads to persistent ER stress, resulting in increased expression of CHOP and subsequent cell death.

Immunohistochemical analysis of GRP78 and CHOP distribution. As shown in Fig. 4, the immunohistochemical investigation revealed the arrest of alveolar septation, characterized as enlarged alveoli and a lack of homogenous small and middle-sized alveoli, in the lung tissues from rats exposed to hyperoxia, compared with those from rats exposed to normal air. GRP78 and CHOP were abundantly expressed in the hyperoxia-exposed rat tissues, whereas the control rats exhibited modest or weak immunoreactivity for these molecules. GRP78 and CHOP were preferentially localized in the alveolar and bronchiolar epithelial cells. These results suggested that hyperoxia increased GRP78 and CHOP content, predominantly in the enlarged and simplified alveolar epithelium.

Localization of apoptosis using a TUNEL assay. The present study determined whether hyperoxia exposure induces CHOP-mediated apoptosis in the lung. Apoptosis was analyzed using a TUNEL assay following exposure to hyperoxia or room air for 14 days. As shown in Fig. 5, apoptosis was induced in the lungs of the rats exposed to hyperoxic conditions. A few TUNEL-positive cells were observed in the control group, however, numerous positive cells were observed in the hyperoxia group. The increase in the number of apoptotic cells paralleled with the increase in GRP78 and CHOP-positive cells, which further indicated that activation of the ER stress pathway, involving CHOP, led to cellular apoptosis in the rat lungs following hyperoxic exposure. 


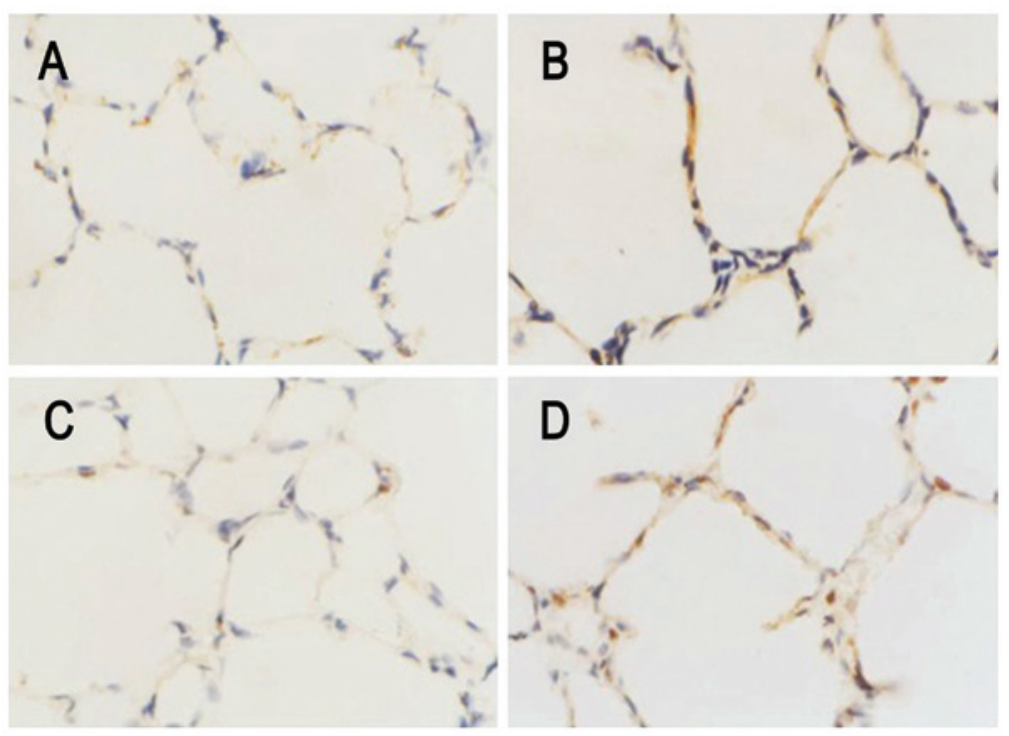

Figure 4. Detection of the expression of GRP78 and CHOP using immunohistochemical staining of rat lungs exposed to room air or hyperoxia for 14 days (magnification, x400). (A) Expression of GRP78 in the control group; (B) Expression of GRP78 in the hyperoxia group; (C) Expression of CHOP in the control group; (D) Expression of CHOP in the hyperoxia group. The results revealed that the expression of GRP78 and CHOP were abundant in the alveolar and bronchiolar epithelial cells of the rats exposed to hyperoxia, while the control rats exhibited weak immunoreactivity for these molecules. GRP78, 78-kDa glucose-regulated/binding immunoglobulin protein; CHOP, CCAAT/enhancer binding protein homologous protein.
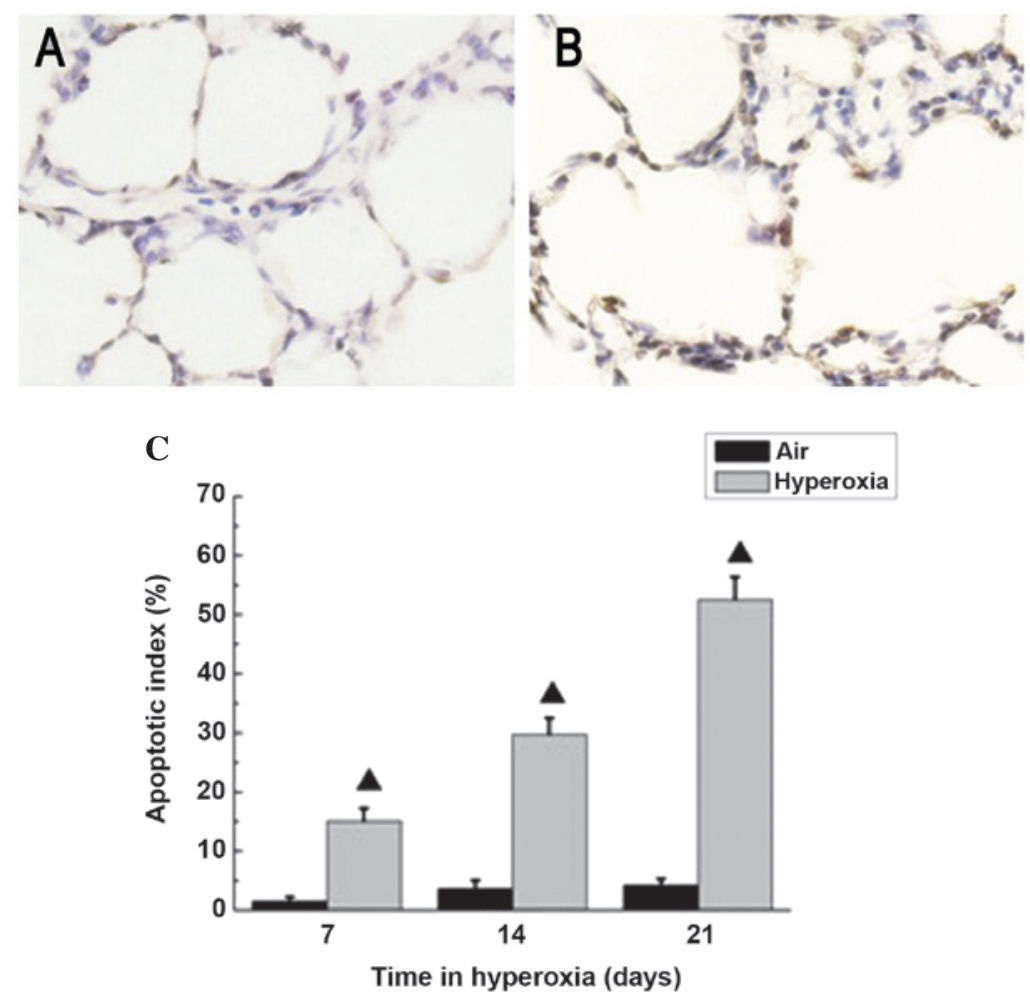

Figure 5. (A and B) TUNEL staining demonstrated a significant increase in TUNEL-positive cells, in a pattern similar to that observed by immunohistochemical staining at 14 days (magnification, x400). (A) Control group; (B) Hyperoxia group. Increased numbers of epithelial and endothelial cells were apoptotic in the hyperoxia group, compared with the control group. (C) Apoptotic cell indices of neonatal rats were increased gradually over the duration of hyperoxia exposure, and the percentages of apoptotic cells were significantly higher, compared with the control group. Data are expressed as the mean \pm standard deviation. ${ }^{\Delta} \mathrm{P}<0.05$, compared with the control. TUNEL, terminal deoxynucleotidyl transferase-mediated dUTP nick end labeling.

\section{Discussion}

Infants born prematurely are at substantial risk for the development of BPD (18). The pathogenesis of BPD remains to be fully elucidated, and multiple contributing injurious factors are considered to be involved. Previous studies have demonstrated that rat alveolar development comprises four stages; pseudoglandular, canalicular, saccular and alveolar; suggesting that the process of alveolar development has a time sequence in the rat is similar to that in humans. The alveolar 
stage (5-21 days gestation) is a key period of lung development, which is particularly affected by mechanical factors. Oxygen toxicity caused by oxygen therapy/hyperoxia is considered to be one of the major contributing factors $(1,19)$. In the present study, it was demonstrated that prolonged exposure to hyperoxia disrupted postnatal alveolarization and led to the development of BPD in rats.

Hyperoxic therapy produces ROS, which can induce apoptosis in the lungs of neonatal rats (3). In the present study, the results of the TUNEL staining in the lung cells suggested that a higher proportion of epithelial and endothelial cells were apoptotic in the BPD model rat lungs, compared with the control group lungs. These results demonstrated that lung cells may undergo increased apoptosis, and apoptotic cell death may be important in hyperoxia-induced lung injury and, therefore, in the development of BPD.

Emerging data have revealed that oxidative stress-induced ER stress may be crucial in the regulation of apoptotic cell death $(20,21)$. ER stress occurs when proteins become misfolded, as can occur with increased oxidative stress or when the capacity of the ER is exceeded by new protein synthesis $(20,22,23)$. Lung epithelial cells secrete a significant quantity of protein, including surfactants, and these cells may be prone to ER stress. In previous investigations, ER stress was reported to be involved in the pathogenesis of several diseases, including in the lungs of lipopolysaccharide (LPS)-treated mice, chronic obstructive pulmonary disease and idiopathic pulmonary fibrosis (24-26). It was also found that CHOP, an apoptosis-associated molecule, is critical in LPS-induced lung damage $(24,27)$. The present study hypothesized that ER stress is also associated with hyperoxia-induced BPD. To investigate this hypothesis, the expression levels of GRP78 and $\mathrm{CHOP}$ were examined in lung cells of a hyperoxia-induced preterm newborn rat BPD model. The results of the present study revealed that, following exposure of the rats to $85 \%$ hyperoxia, the expression of GRP78 in the lung tissues increased gradually at 7, 14 and 21 days, and the expression levels were significantly higher than those in the control air group. GRP78 is a central regulator of ER homeostasis, and its upregulation is widely used as a sentinel marker for ER stress under pathological conditions $(28,29)$. Therefore, increased expression of GRP78, which indicated ER stress, was induced in hyperoxia-induced BPD.

GRP78 facilitates protein folding, preventing intermediates from aggregating and promoting the proteasomal degradation of misfolded proteins $(6,28)$. However, if ER homeostasis cannot be restored, a prolonged ER stress response may induce apoptosis. CHOP is critical in ER stress-induced apoptosis $(5,6)$. The induction of CHOP has previously been reported in the lungs of mice exposed to hyperoxia, with immunohistochemical and in situ hybridization investigations localizing its expression predominantly to the bronchiolar epithelium and, to a lesser extent, throughout the lung parenchyma (30). The present study found that persistent hyperoxic exposure upregulated the expression of $\mathrm{CHOP}$ and also demonstrated that the enhanced expression levels of GRP78 and CHOP were associated with an increase in the number of apoptotic cells. These results suggested a crucial role for CHOP in the apoptosis in hyperoxia-induced BPD.
The present study demonstrated that hyperoxia increased the expression of CHOP in the lung and that CHOP was involved in the ER stress-mediated apoptosis pathway, accelerating the process of hyperoxia-induced lung injury. In contrast to these findings, Lozon et al (31) observed that the increase in the expression of CHOP was independent of ER stress and conferred protection, rather than increased susceptibility, to hyperoxia-induced lung injury. Differences between the two studies included the use of preterm rats, rather than adult mice, in the present study, and the animal model of hyperoxic lung injury in the present study was established following a longer duration of exposure to oxygen. Therefore, the present study hypothesized that the ER stress pathway, involving CHOP, and ER stress-mediated apoptosis were involved in the pathogenesis of BPD. Several studies have demonstrated that decreasing the levels of the anti-apoptotic facto, B-cell lymphoma 2, and increasing the levels of ROS are important in the activation of CHOP $(21,32)$. Therefore, further investigation of the precise mechanism of ER stress-mediated apoptosis downstream of CHOP induction is required.

In conclusion, the present study demonstrated that exposure to $85 \%$ hyperoxia induced the expression of CHOP in preterm rats by a mechanism dependent on the ER stress pathway. Thus, the activation of CHOP may be involved in the pathogenesis of BPD under conditions of hyperoxic stress or inflammatory responses. Whether inhibition of the protein expression of $\mathrm{CHOP}$ is protective for hyperoxia-induced lung injury remains to be elucidated.

\section{Acknowledgements}

The present study was supported by the National Natural Science Foundation of China (grant no. 81370746), the Natural Science Foundation of Jiangsu Province, China (grant no. BK2011485) and the Social Development Foundation of Zhenjiang, China (grant no. SH2011022).

\section{References}

1. Jobe AH and Bancalari E: Bronchopulmonary dysplasia. Am J Respir Crit Care Med 163: 1723-1729, 2001

2. Chen Y, Martinez MA and Frank L: Prenatal dexamethasone administration to premature rats exposed to prolonged hyperoxia: A new rat model of pulmonary fibrosis (bronchopulmonary dysplasia). J Pediatr 130: 409-416, 1997.

3. Zhang X, Shan P, Sasidhar M, Chupp GL, Flavell RA, Choi AM and Lee PJ: Reactive oxygen species and extracellular signal-regulated kinase $1 / 2$ mitogen-activated protein kinase mediate hyperoxia-induced cell death in lung epithelium. Am J Respir Cell Mol Biol 28: 305-315, 2003.

4. Szegezdi E, Logue SE, Gorman AM and Samali A: Mediators of endoplasmic reticulum stress-induced apoptosis. EMBO Rep 7: $880-885,2006$

5. Malhotra JD and Kaufman RJ: The endoplasmic reticulum and the unfolded protein response. Semin Cell Dev Biol 18: 716-731, 2007.

6. Ron D and Walter P: Signal integration in the endoplasmic reticulum unfolded protein response. Nat Rev Mol Cell Biol 8: 519-529, 2007.

7. Lai E, Teodoro T and Volchuk A: Endoplasmic reticulum stress: Signaling the unfolded protein response. Physiology (Bethesda) 22: 193-201, 2007.

8. Zhang K and Kaufman RJ: Signaling the unfolded protein response from the endoplasmic reticulum. J Biol Chem 279: 25935-25938, 2004.

9. Marciniak SJ, Yun CY, Oyadomari S, Novoa I, Zhang Y, Jungreis R, Nagata K, Harding HP and Ron D: CHOP induces death by promoting protein synthesis and oxidation in the stressed endoplasmic reticulum. Genes Dev 18: 3066-3077, 2004. 
10. Zinszner H, Kuroda M, Wang X, Batchvarova N, Lightfoot RT, Remotti H, Stevens JL and Ron D: CHOP is implicated in programmed cell death in response to impaired function of the endoplasmic reticulum. Genes Dev 12: 982-995, 1998.

11. Oyadomari S and Mori M: Roles of CHOP/GADD153 in endoplasmic reticulum stress. Cell Death Differ 11: 381-389, 2004.

12. Song B, Scheuner D, Ron D, Pennathur S and Kaufman RJ: Chop deletion reduces oxidative stress, improves beta cell function, and promotes cell survival in multiple mouse models of diabetes. J Clin Invest 118: 3378-3389, 2008.

13. Tessitore A, del P Martin M, Sano R, Ma Y, Mann L, Ingrassia A, Laywell ED, Steindler DA, Hendershot LM and d'Azzo A: GM1-ganglioside-mediated activation of the unfolded protein response causes neuronal death in a neurodegenerative gangliosidosis. Mol Cell 15: 753-766. 2004.

14. Yuan Y, Guo Q, Ye Z, Pingping X, Wang N and Song Z: Ischemic postconditioning protects brain from ischemia/reperfusion injury by attenuating endoplasmic reticulum stress-induced apoptosis through PI3K-Akt pathway. Brain Res 1367: 85-93, 2011.

15. Miyazaki Y, Kaikita K, Endo M, Horio E, Miura M, Tsujita K, Hokimoto S, Yamamuro M, Iwawaki T, Gotoh T, et al: C/EBP homologous protein deficiency attenuates myocardial reperfusion injury by inhibiting myocardial apoptosis and inflammation. Arterioscler Thromb Vasc Biol 31: 1124-1132, 2011.

16. Emery JL and Mithal A: The number of alveoli in the terminal respiratory unit of man during late intrauterine life and childhood. Arch Dis Child 35: 544-547, 1960.

17. Coalson JJ: Pathology of new bronchopulmonary dysplasia. Semin Neonatal 8: 73-81, 2003.

18. Merritt TA, Deming DD and Boynton BR: The 'new' bronchopulmonary dysplasia: Challenges and commentary. Semin Fetal Neonatal Med 14: 345-357, 2009.

19. Saugstad OD: Oxygen and oxidative stress in bronchopulmonary dysplasia. J Perinat Med 38: 571-577, 2010.

20. Malhotra JD and Kaufman RJ: Endoplasmic reticulum stress and oxidative stress: A vicious cycle or a double-edged sword? Antioxid Redox Signal 9: 2277-2293, 2007.

21. Tabas I and Ron D: Integrating the mechanisms of apoptosis induced by endoplasmic reticulum stress. Nat Cell Biol 13: 184-190, 2011
22. Shore GC, Papa FR and Oakes SA: Signaling cell death from the endoplasmic reticulum stress response. Curr Opin Cell Biol 23: 143-149, 2011.

23. Jäger R, Bertrand MJ, Gorman AM, Vandenabeele P and Samali A: The unfolded protein response at the crossroads of cellular life and death during endoplasmic reticulum stress. Biol Cell 104: 259-270, 2012.

24. Endo M, Oyadomari S, Suga M, Mori M and Gotoh T: The ER stress pathway involving CHOP is activated in the lungs of LPS-treated mice. J Biochem 138: 501-507, 2005.

25. Greene CM and McElvaney NG: Protein misfolding and obstructive lung disease. Proc Am Thorac Soc 7: 346-355, 2010.

26. Korfei M, Ruppert C, Mahavadi P, Henneke I, Markart P, Koch M, Lang G, Fink L, Bohle RM, Seeger W, et al: Epithelial endoplasmic reticulum stress and apoptosis in sporadic idiopathic pulmonary fibrosis. Am J Respir Crit Care Med 178: 838-846, 2008.

27. Nakayama Y, Endo M, Tsukano H, Mori M, Oike Y and Gotoh T: Molecular mechanisms of the LPS-induced non-apoptotic ER stress-CHOP pathway. J Biochem 147: 471-483, 2010.

28. Rao RV, Peel A, Logvinova A, del Rio G, Hermel E, Yokota T, Goldsmith PC, Ellerby LM, Ellerby HM and Bredesen DE: Coupling endoplasmic reticulum stress to the cell death program: Role of the ER chaperone GRP78. FEBS Lett 514: 122-128, 2002.

29. Lee AS: The ER chaperone and signaling regulator GRP78/BiP as a monitor of endoplasmic reticulum stress. Methods 35: 373-381, 2005.

30. O'Reilly MA, Staversky RJ, Watkins RH, Maniscalco WM and Keng PC: p53-independent induction of GADD45 and GADD153 in mouse lungs exposed to hyperoxia. Am J Physiol Lung Cell Mol Physiol 278: L552-L559, 2000.

31. Lozon TI, Eastman AJ, Matute-Bello G, Chen P, Hallstrand TS and Altemeier WA: PKR-dependent CHOP induction limits hyperoxia-induced lung injury. Am J Physiol Lung Cell Mol Physiol 300: L422-L429, 2011.

32. Sung B, Ravindran J, Prasad S, Pandey MK and Aggarwal BB: Gossypol induces death receptor-5 through activation of the ROS-ERK-CHOP pathway and sensitizes colon cancer cells to TRAIL. J Biol Chem 285: 35418-35427, 2010 